\title{
Endoscopic Ultrasound-Guided Biliary Access, with Focus on Technique and Practical Tips
}

\author{
Woo Hyun Paik ${ }^{1}$ and Do Hyun Park ${ }^{2}$ \\ ${ }^{1}$ Department of Internal Medicine and Liver Research Institute, Seoul National University Hospital, Seoul National University College of \\ Medicine, Seoul, ${ }^{2}$ Division of Gastroenterology, Department of Internal Medicine, Asan Medical Center, University of Ulsan College of \\ Medicine, Seoul, Korea
}

In 1980, endoscopic ultrasound (EUS) was introduced as a diagnostic tool for evaluation of the pancreas. Since the introduction of curvilinear-array echoendoscopy, EUS has been used for a variety of gastrointestinal interventions, including fine needle aspiration, tumor ablation, and pancreatobiliary access. One of the main therapeutic roles of EUS is biliary drainage as an alternative to endoscopic retrograde biliary drainage (ERBD) or percutaneous transhepatic biliary drainage (PTBD). This article summarizes three different methods of EUS-guided biliary access, with focus on technique and practical tips. Clin Endosc 2017;50:104-111

Key Words: Endosonography; Cholestasis; Biliary drainage; Cholangiopancreatography, endoscopic retrograde; Percutaneous transhepatic biliary drainage

\section{INTRODUCTION}

Endoscopic retrograde cholangiopancreatography (ERCP) and endoscopic retrograde biliary drainage (ERBD) are firstline, non-surgical interventions for biliary obstruction, with a success rate of approximately $90 \%-97 \%$, and a less than $10 \%$ risk of adverse events. ${ }^{1,2}$ However, ERCP may not achieve biliary decompression when surgically altered anatomy or duodenal obstruction is present. ${ }^{3,4}$ Percutaneous transhepatic biliary drainage (PTBD) is a rescue procedure used after failed ERCP. ${ }^{5}$ The technical success rate of PTBD is reportedly more than 95\%; the adverse events associated with PTBD are bleeding, infection, dislodgement, bile leak, and tract seeding, with overall rates of $33 \%$ or higher. ${ }^{6,7}$ Although PTBD is an es-

Received: March 4, 2017 Accepted: March 12, 2017

Correspondence: Do Hyun Park

Division of Gastroenterology, Department of Internal Medicine, Asan Medical Center, University of Ulsan College of Medicine, 88 Olympic-ro 43-gil, Songpa-gu, Seoul 05505, Korea

Tel: +82-2-3010-3194, Fax: +82-2-3010-8043, E-mail: dhpark@amc.seoul.kr

(cc) This is an Open Access article distributed under the terms of the Creative Commons Attribution Non-Commercial License (http://creativecommons.org/ licenses/by-nc/3.0) which permits unrestricted non-commercial use, distribution, and reproduction in any medium, provided the original work is properly cited. tablished rescue procedure for ERCP, PTBD is uncomfortable to the patient because of the external drainage catheter, and is not suitable when ascites or multiple liver metastasis are present. $^{7}$ Since endoscopic ultrasound (EUS)-guided cholangiography was first described in 1996, ${ }^{8}$ EUS-guided biliary drainage (EUS-BD) has been an evolving alternative to PTBD, ${ }^{4,-11}$ with advantages over PTBD that include drainage internally and a single session procedure by the same operator, even after failed ERCP. However, EUS-BD still carries some risk and is only performed in a limited number of centers. ${ }^{12}$ EUS-BD can be performed by three methods: EUS-guided rendezvous technique, EUS-guided transmural stenting, and EUS-guided antegrade stenting. ${ }^{9}$ EUS-guided biliary access (except for the gallbladder) and its clinical applications will be reviewed, with a focus on technique and practical tips, and ERBD, PTBD, and EUS-BD will be compared.

\section{PROTOCOL AND TECHNICAL TIPS FOR EUS-BD}

\section{EUS-guided rendezvous technique}

EUS-guided rendezvous technique is usually considered 

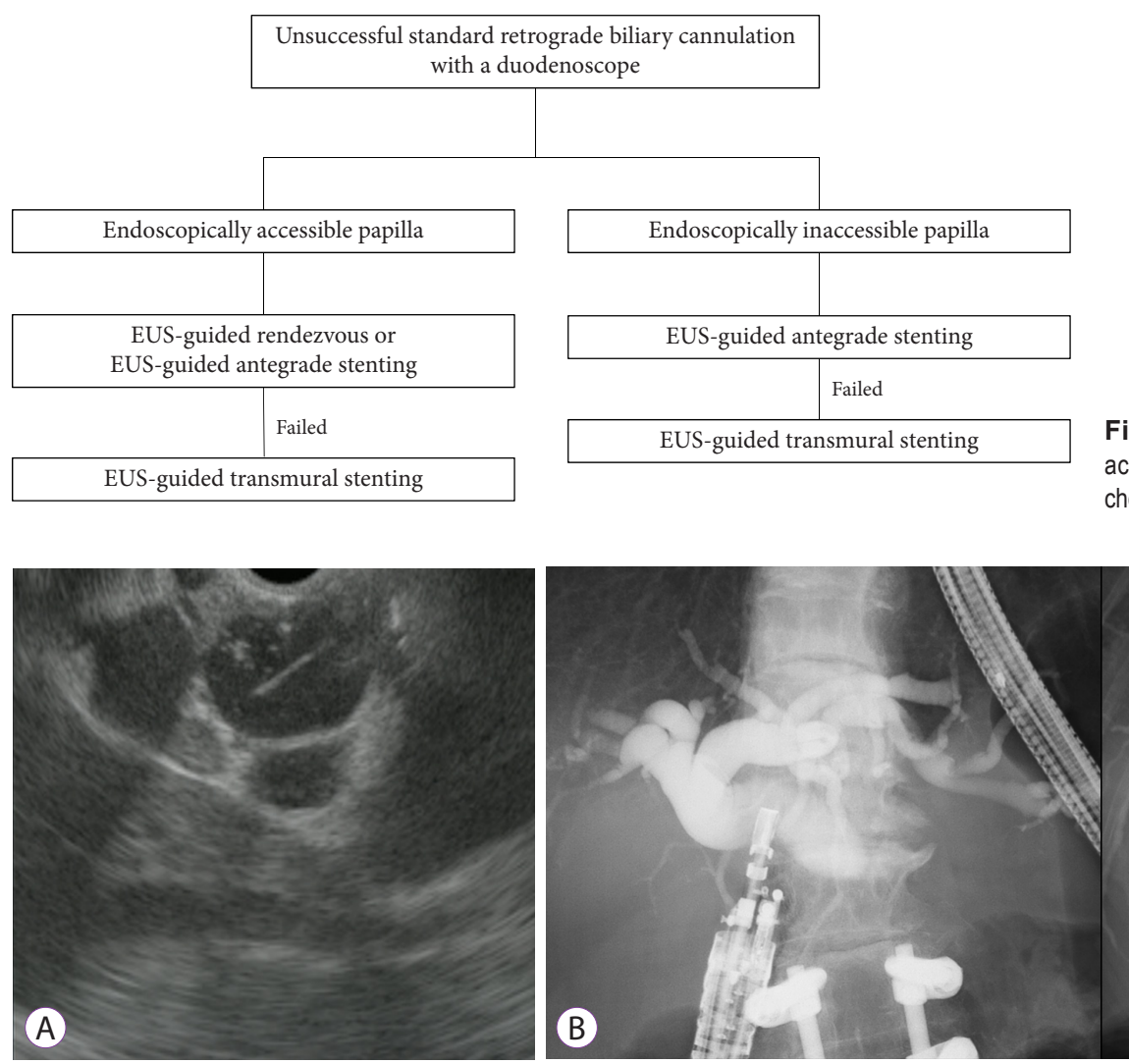

Fig. 1. Endoscopic ultrasound (EUS)-guided, biliary access algorithm after failed endoscopic retrograde cholangiopancreatography (ERCP).
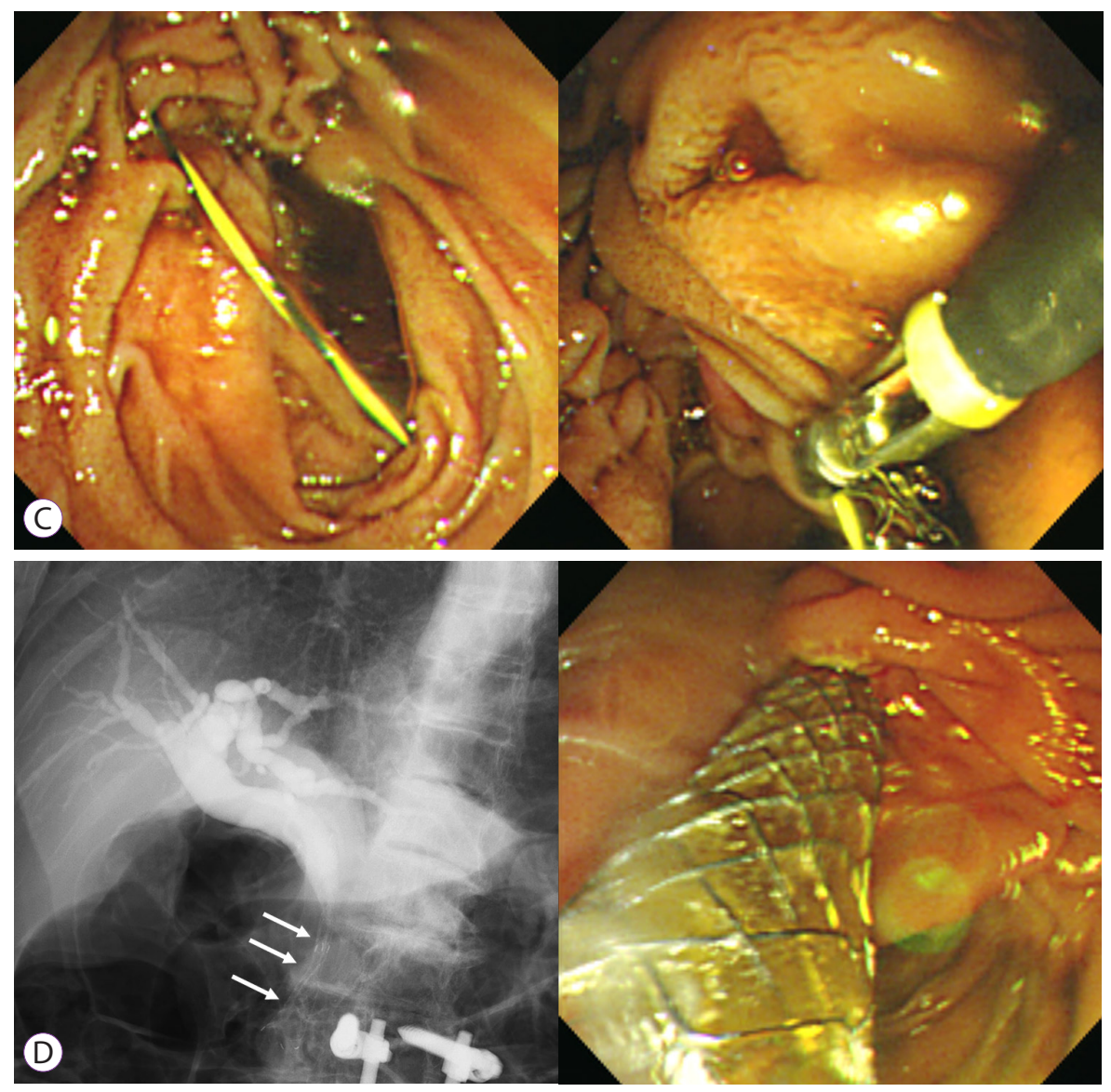

Fig. 2. Endoscopic ultrasound (EUS)-guided rendezvous therapy was performed in an 82-year-old female with malignant distal biliary obstruction due to pancreatic cancer. (A) The dilated common bile duct was punctured at the duodenal bulb with a 19-gauge EUS needle. (B) Contrast was injected under fluoroscopy, and a guidewire was manipulated to pass across the ampulla and looped inside the duodenum. (C) The looped guidewire was grasped with a rat tooth forceps, and pulled through the working channel. (D) A metal stent (white arrows) was inserted in retrograde manner. 
when selective cannulation of the bile duct fails with ERCP (Fig. 1). ${ }^{13}$ Moreover, EUS-guided rendezvous technique was found to be better than precut papillotomy for single session biliary access in a retrospective nonrandomized study. ${ }^{14}$ The extra- or intrahepatic ducts are usually accessed with a 19-gauge EUS needle under EUS guidance. ${ }^{15}$ The extrahepatic approach is preferred to the intrahepatic approach because the diameter of the extrahepatic ducts is larger and the distance between the biliary access site and the ampulla is shorter. ${ }^{15}$ After confirming access of the bile duct by aspiration of bile juice, contrast is injected into the bile duct for cholangiography. Then, a guidewire negotiates a stricture site and passes across the papilla in an antegrade manner; subsequent retrograde cannulation of the bile duct is performed as conventional ERCP. A couple of loops of guidewire should be placed inside the small intestine to maintain stability during removal of the EUS needle and endoscope. ${ }^{14}$ Selective bile duct cannulation following the guidance of the existing guidewire is favored, because it requires less effort and time. However, when the above method is not possible, the coiled guidewire is grasped with a rat tooth forceps or snare, and then the guidewire is pulled through the working channel (Fig. 2). EUS-guided rendezvous technique may be the safest method of EUS-guided biliary access, since it preserves normal anatomy without fistula dilation. ${ }^{16}$ However, EUS-guided rendezvous technique is limited to when the papilla is accessible endoscopically. ${ }^{9,14}$ Moreover, guidewire manipulation to pass through the papilla is mandatory, and procedural time may be prolonged, since change of the endoscope is required during the procedure. Therefore, the success rate of EUS-guided rendezvous technique is reported to be somewhat lower than that of EUS-guided transmural stenting, ranging from 35\%$100 \% .{ }^{15}$ The most common reason for failure of EUS-guided rendezvous technique is inability to manipulate the guidewire through the papilla and endoscopically inaccessible papilla. ${ }^{15}$ A recent meta-analysis of EUS-BD techniques reported that technical success and adverse event rates using EUS-guided rendezvous technique were 89.7\% (104/116) and 13.3\% (2/15), respectively; however, available data for EUS-guided rendezvous technique were insufficient in this meta-analysis. ${ }^{17}$
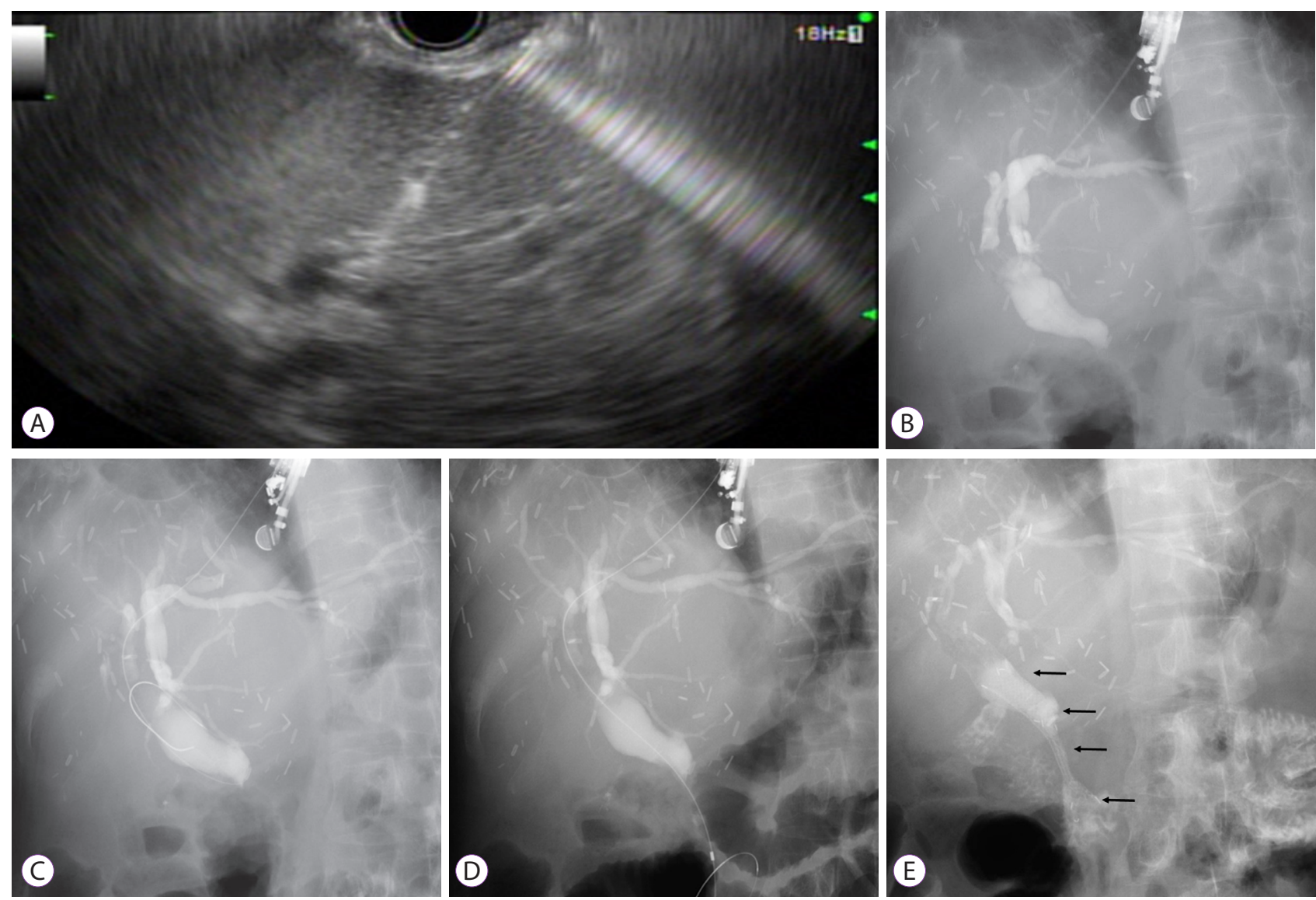

Fig. 3. Endoscopic ultrasound (EUS)-guided antegrade stenting was performed in a 47-year-old female with malignant distal biliary obstruction due to advanced gastric cancer. The ampulla was not accessible due to previous total gastrectomy with Roux-en-Y anastomosis. (A) The dilated intrahepatic bile duct segment 2 was punctured with a 19-gauge EUS needle. (B) Contrast was injected for cholangiography. (C) A guidewire was placed and coiled inside the common bile duct. (D) A guidewire was manipulated with a 4-F catheter to pass across the papilla. (E) An uncovered metal stent was inserted in antegrade manner (black arrows). 


\section{EUS-guided antegrade stenting}

A 19-gauge EUS needle is used to access the biliary system, and puncture of the intrahepatic bile duct segment two (B2) is preferred, since B2 is likely to present as a straight line, which facilitates a guidewire approach into the hilum. ${ }^{2}$ During guidewire manipulation to pass across the hilum and negotiate the stricture site, the EUS needle is withdrawn; a 4-F cannula is then used to advance a guidewire across the fistula and into the intrahepatic ducts, since the guidewire may be torn off by the cutting tip of an EUS needle. ${ }^{9}$ Finally, a metal stent is placed along the guidewire in an antegrade manner
(Fig. 3). A 4-mm balloon catheter can be used for dilation of the papilla and intrahepatic ducts to facilitate advancement of the stent delivery catheter. ${ }^{9}$

An enhanced guidewire manipulation protocol for EUS$\mathrm{BD}$ had been proposed, and EUS-guided rendezvous and antegrade stenting should be considered before EUS-guided transmural stenting. ${ }^{9}$ EUS-guided antegrade stenting has an advantage over rendezvous technique, because exchange of the endoscope is not required during the procedure. Furthermore, EUS-guided antegrade stenting can be achieved even when the papilla is inaccessible. The technical success and
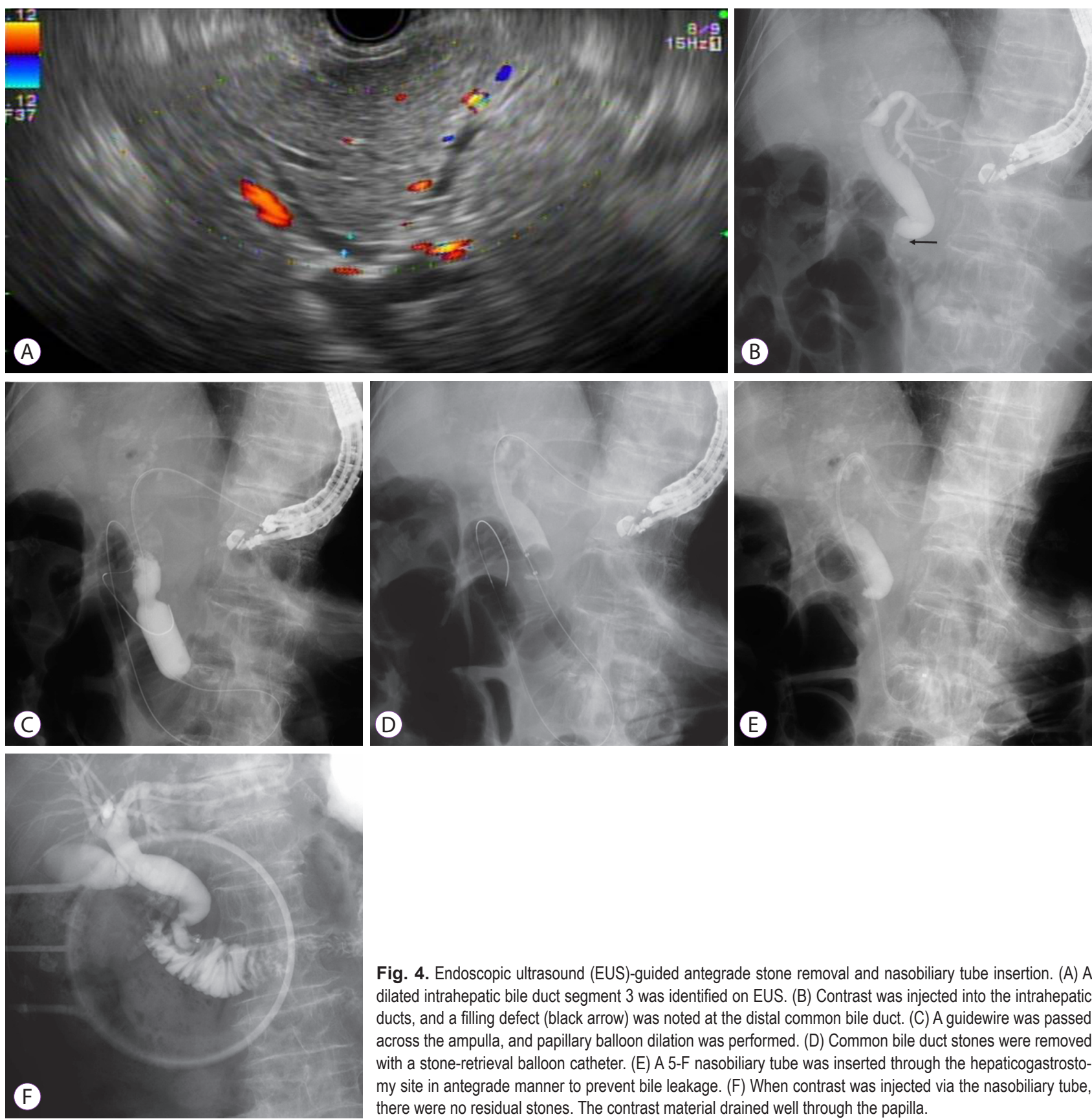

Fig. 4. Endoscopic ultrasound (EUS)-guided antegrade stone removal and nasobiliary tube insertion. (A) A dilated intrahepatic bile duct segment 3 was identified on EUS. (B) Contrast was injected into the intrahepatic ducts, and a filling defect (black arrow) was noted at the distal common bile duct. (C) A guidewire was passed across the ampulla, and papillary balloon dilation was performed. (D) Common bile duct stones were removed with a stone-retrieval balloon catheter. (E) A 5-F nasobiliary tube was inserted through the hepaticogastrostomy site in antegrade manner to prevent bile leakage. (F) When contrast was injected via the nasobiliary tube, there were no residual stones. The contrast material drained well through the papilla. 
adverse event rates of EUS-guided antegrade stenting were 91.3\% (42/46) and 33.3\% (2/6) in a meta-analysis; however, this result is inconclusive due to the small sample size. ${ }^{17}$

When the papilla is inaccessible endoscopically in patients with common bile duct stones, a percutaneous or surgical approach is considered first. In this situation, EUS-guided antegrade stone removal can be effective and more comfortable for patients, since PTBD and subsequent tract dilation are not required (Fig. 4).

\section{EUS-guided transmural stenting}

EUS-guided transmural stenting is usually classified as EUS-guided choledochoduodenostomy (EUS-CDS) and EUS-guided hepaticogastrostomy (EUS-HGS). Occasionally, EUS-guided choledochoantrostomy or hepaticoduodenostomy is also performed. ${ }^{18-21}$ The procedure of EUS-guided transmural stenting is composed of complex steps as follows: biliary access with EUS needle, guidewire manipulation, fistula dilation, and stent placement (Fig. 5). The optimal access point for EUS-HGS is a significant technical concern, and a bile duct diameter more than $5 \mathrm{~mm}$ and hepatic segment length of 1-3 $\mathrm{cm}$ on EUS may be required for successful EUS-HGS. ${ }^{22}$ When accessing the intrahepatic ducts for puncture, B3 is preferred over $\mathrm{B} 2$ because $\mathrm{B} 3$ puncture is usually made in the stomach body lesser curvature; thus, during deployment, the tip of the stent in the stomach can be verified, and adverse events such as stent migration can be prevented. ${ }^{2,23}$ However, B2 puncture can sometimes be made via the transesophageal route, and a risk of severe adverse events such as mediastinitis can develop. ${ }^{24}$ It is important to attach the probe of the echoendoscope to the duodenal or gastric mucosa during EUS-BD. In EUS$\mathrm{CDS}$, the push method is preferred to the pull method because the position of the endoscope is stabilized and the EUS needle is directed toward the hilar portion. ${ }^{25}$ In EUS-HGS, to stabilize the location of the echoendoscope in the stomach high body or cardia during stent delivery, the front one-half of a metal stent is deployed first under EUS and fluoroscopic guidance. Then, when deploying the remaining metal stent within the working channel of the endoscope under fluoroscopic guidance, the endoscope is pulled out cautiously. ${ }^{26}$ During guidewire manipulation, inordinate force should be avoided to prevent tearing or peeling of the guidewire. When the guidewire is inadvertently advanced into the peripheral bile duct, use of a liver impaction technique that withdraws the EUS needle into the hepatic parenchyma can prevent guidewire tearing. ${ }^{27}$ Most adverse events during EUS-guided transmural stenting
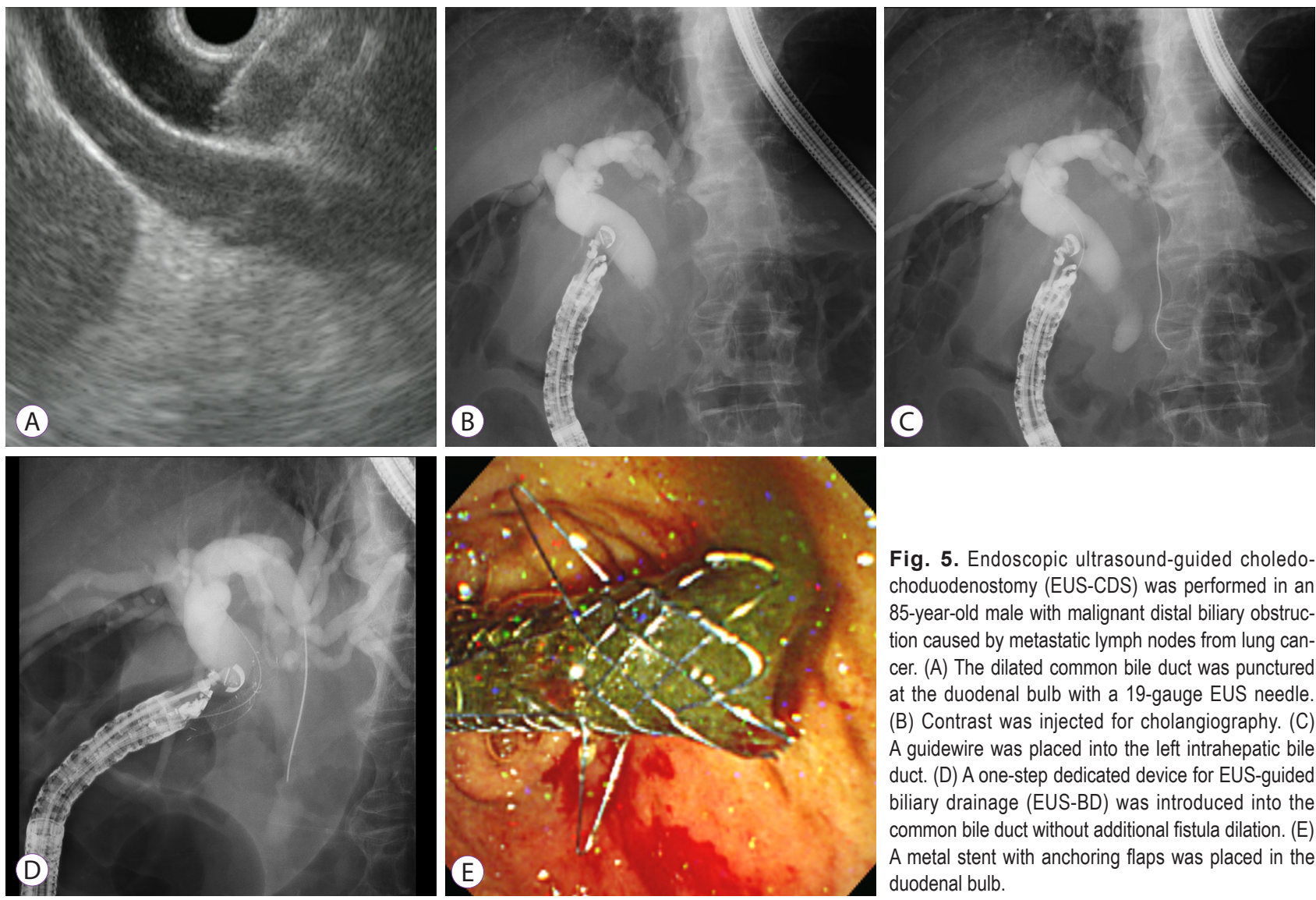

Fig. 5. Endoscopic ultrasound-guided choledochoduodenostomy (EUS-CDS) was performed in an 85-year-old male with malignant distal biliary obstruction caused by metastatic lymph nodes from lung cancer. (A) The dilated common bile duct was punctured at the duodenal bulb with a 19-gauge EUS needle. (B) Contrast was injected for cholangiography. (C) A guidewire was placed into the left intrahepatic bile duct. (D) A one-step dedicated device for EUS-guided biliary drainage (EUS-BD) was introduced into the common bile duct without additional fistula dilation. (E) A metal stent with anchoring flaps was placed in the duodenal bulb. 
develop during fistula dilation. ${ }^{12,28}$ Sequential fistula dilation with a bougie catheter may be preferred over cautery dilation because of safety issues. ${ }^{26,29}$ However, sequential dilation generates axial force, and may cause separation between the liver and stomach or the common bile duct and duodenum. ${ }^{30}$ To prevent the separation of tissue planes, a balloon catheter can be used for fistula dilation. Since a balloon is inserted in a compressed state, the diameter of the delivery catheter can be minimized to about $5 \mathrm{~F}$. Furthermore, a balloon catheter generates radial force during fistula dilation. ${ }^{30}$ However, a recent meta-analysis of EUS-BD technique reported adverse event rates for each dilation device without statistical comparison as follows: needle knife, $20 \%$ (49/249) vs. balloon catheter, $20.4 \%$ $(44 / 216)$ vs. cystotome, $38.5 \%(10 / 26){ }^{17}$

Metal stents are preferred over plastic stents for EUS-guided transmural stenting, ${ }^{31}$ and a dedicated stent introducer for EUS-BD was recently developed..$^{28}$ This one-step dedicated device for EUS-BD has a fine metal tip and a 7-F shaft that enables stent insertion without additional fistula dilation, and a modified hybrid metal stent with anchoring flaps and uncovered portion is loaded in the intrahepatic ducts to prevent stent migration. Compared to a conventional stent delivery system with a fully covered metal stent, this novel device showed similar efficacy and safety with less chance of extra fistula dilation, which enables a shorter procedure time. ${ }^{28}$

During EUS-HGS, the length of a stent should be sufficient to prevent proximal migration into the peritoneum. ${ }^{2}$ To maintain stent patency, a length more than $3 \mathrm{~cm}$ in the gastric portion is recommended for EUS-HGS. ${ }^{32}$ We suggest the following formula to determine the length of a stent: the distance $(\mathrm{cm})$ of an EUS needle between the stomach wall and accessed left intrahepatic duct on EUS (e.g., approximately half of a stent in the hepatic parenchyma) multiplied by two (e.g., remaining half of a stent deployed inside the endoscope, including possible stent shortening) plus $1 \mathrm{~cm}$ (e.g., the intrahepatic portion of a metal stent). ${ }^{26}$

In 29 studies of EUS-guided transmural stenting, the technical and functional success rates for EUS-guided transmural stenting were $95.7 \%$ (409/532) and 90.3\% (401/444), respectively. ${ }^{17}$ The overall adverse event rate was $24.4 \%$ (114/467), and the most common adverse events were stent migration (5.4\%), penumoperitoneum (3.4\%), peritonitis (3\%), bleeding $(2.8 \%)$, cholangitis (3\%), and bile leakage $(1.5 \%) .^{17}$ There was no significant difference in procedure-related adverse events between EUS-HGS and EUS-CDS (25\% vs. $21.8 \%, p=0.465){ }^{17}$ However, in cases of biliary obstruction combined with duodenal obstruction, EUS-HGS might be superior to EUS-CDS, with longer stent patency and fewer adverse events due to duodenobiliary reflux. ${ }^{33}$

EUS-HGS may not be feasible in patients with advanced hi- lar obstruction, when the intrahepatic bile ducts are separated. Segment 2 or 3 left intrahepatic ducts are generally selected for puncture in EUS-HGS; thus, right-sided intrahepatic biliary obstruction was considered less effective for biliary drainage. ${ }^{2}$ However, novel techniques of right lobe drainage with EUS-HGS as a bridging method (uncovered metal stent between right and left intrahepatic ducts; covered metal stent between left intrahepatic duct and stomach) or hepaticoduodenostomy have been tried recently, ${ }^{21,34}$ and these approaches could make EUS-HGS effective in advanced hilar obstruction.

\section{Learning curve for EUS-BD}

The clinical application of EUS-BD is increasing; however, when performing EUS-BD, the technical success rate is lower and the adverse event rate is higher than in previously published data. ${ }^{12}$ To date, no systematic EUS-BD training program has been established, and regular practice dedicated to EUS-BD is difficult. ${ }^{30}$ Moreover, dedicated devices and accessories for EUS-BD are lacking. Although there is no standard recommendation for the number of procedures needed prior to attempting EUS-BD, a recent consortium meeting of experts suggested the following: (1) performance of many ERCP procedures with a success rate greater than 95\%; (2) performance of many routine pancreatobiliary EUS-guided and fine needle aspiration procedures; (3) support of interventional radiologists and pancreatobiliary surgeons. ${ }^{30} \mathrm{~A}$ recent, single-center experience with EUS-HGS revealed that over 33 cases might be required to achieve a plateau phase for successful EUS-HGS. ${ }^{22}$ EUS-BD assisted by preexisting PTBD could provide an additional advantage for advanced endoscopists early on the learning curve for EUS-guided biliary access. ${ }^{35}$ By opacification of the intrahepatic duct via a PTBD tube before puncturing with an EUS needle, mistakes during EUS-BD could be prevented. Even if EUS-BD fails, the risk of adverse events, including cholangitis and bile leakage, would decrease because of the indwelling PTBD catheter.

\section{Comparison between PTBD, ERBD, and EUS-BD}

In comparing these biliary drainage methods, technical feasibility, safety, cost, comfort, compliance, and duration are important issues. A previous retrospective study comparing PTBD $(n=12)$ and EUS-CDS ( $n=13)$ reported similar success, adverse event rates, cost, and quality of life. ${ }^{36}$ However, since only direct procedural costs were compared between the two groups, this likely overestimates the cost-effectiveness of PTBD, which is associated with increased downstream costs due to the requirement for frequent reinterventions. Another retrospective study comparing PTBD $(n=51)$ and EUS-BD $(n=22)$ also reported similar success and adverse event rates between the two procedures; however, the reintervention 
rates were significantly higher in the PTBD group than in the EUS-BD group ( $80.4 \%$ vs. $15.7 \%, p<0.001) .{ }^{37}$ When considering all subsequent reinterventions, adverse events developed more frequently in the PTBD group. ${ }^{37}$ A recent, multicenter, open-label, prospective, randomized trial comparing EUS$\mathrm{BD}$ with a dedicated one-step device and PTBD for malignant distal biliary obstruction after failed ERCP showed similar efficacy and quality of life; however, fewer procedure-related adverse events and unscheduled reinterventions occurred in the EUS-BD group. ${ }^{11}$ A systematic review also revealed that EUS-BD is associated with significantly better clinical success, a lower rate of adverse events, and fewer reinterventions. ${ }^{38}$ EUS-BD could be a good alternative or might be superior to PTBD after failed ERCP, when a dedicated device for EUS-BD and an experienced operator are available.

A multicenter, prospective, randomized, comparative study of primary ERBD and EUS-BD in patients with malignant distal biliary obstruction is being conducted in Korea. The results of this study could provide future perspectives on EUSBD.

\section{CONCLUSIONS}

EUS is a very attractive tool for accessing the biliary system, not only for diagnosis but also for treatment. EUS-BD is an alternative, minimally invasive technique for use when ERCP fails. However, as a standardized protocol and long-term treatment outcomes for EUS-BD are still under investigation, EUS-BD should be performed by endoscopists with expertise in both ERCP and EUS. Further advances and availability of dedicated devices and accessories may improve the outcomes of EUS-BD.

\section{Conflicts of Interest}

The authors have no financial conflicts of interest.

\section{REFERENCES}

1. Schöfl R. Diagnostic endoscopic retrograde cholangiopancreatography. Endoscopy 2001;33:147-157.

2. Park DH. Endoscopic ultrasonography-guided hepaticogastrostomy. Gastrointest Endosc Clin N Am 2012;22:271-280, ix

3. Coté GA, Singh S, Bucksot LG, et al. Association between volume of endoscopic retrograde cholangiopancreatography at an academic medical center and use of pancreatobiliary therapy. Clin Gastroenterol Hepatol 2012;10:920-924

4. Kedia P, Gaidhane M, Kahaleh M. Endoscopic guided biliary drainage: how can we achieve efficient biliary drainage? Clin Endosc 2013;46:543551

5. Inamdar S, Slattery E, Bhalla R, Sejpal DV, Trindade AJ. Comparison of adverse events for endoscopic vs percutaneous biliary drainage in the treatment of malignant biliary tract obstruction in an inpatient national cohort. JAMA Oncol 2016;2:112-117.

6. Saad WE, Wallace MJ, Wojak JC, Kundu S, Cardella JF. Quality improvement guidelines for percutaneous transhepatic cholangiography, biliary drainage, and percutaneous cholecystostomy. J Vasc Interv Radiol 2010;21:789-795.

7. Nennstiel S, Weber A, Frick G, et al. Drainage-related complications in percutaneous transhepatic biliary drainage: an analysis over 10 years. J Clin Gastroenterol 2015;49:764-770.

8. Wiersema MJ, Sandusky D, Carr R, Wiersema LM, Erdel WC, Frederick PK. Endosonography-guided cholangiopancreatography. Gastrointest Endosc 1996;43(2 Pt 1):102-106.

9. Park DH, Jeong SU, Lee BU, et al. Prospective evaluation of a treatment algorithm with enhanced guidewire manipulation protocol for EUS-guided biliary drainage after failed ERCP (with video). Gastrointest Endosc 2013;78:91-101.

10. Giovannini M, Dotti M, Bories E, et al. Hepaticogastrostomy by echo-endoscopy as a palliative treatment in a patient with metastatic biliary obstruction. Endoscopy 2003;35:1076-1078.

11. Lee TH, Choi JH, Park do H, et al. Similar efficacies of endoscopic ultrasound-guided transmural and percutaneous drainage for malignant distal biliary obstruction. Clin Gastroenterol Hepatol 2016;14:1011-1019. e3.

12. Vila JJ, Pérez-Miranda M, Vazquez-Sequeiros E, et al. Initial experience with EUS-guided cholangiopancreatography for biliary and pancreatic duct drainage: a Spanish national survey. Gastrointest Endosc 2012;76:1133-1141.

13. Sarkaria S, Lee HS, Gaidhane M, Kahaleh M. Advances in endoscopic ultrasound-guided biliary drainage: a comprehensive review. Gut Liver 2013;7:129-136

14. Dhir V, Bhandari S, Bapat M, Maydeo A. Comparison of EUS-guided rendezvous and precut papillotomy techniques for biliary access (with videos). Gastrointest Endosc 2012;75:354-359.

15. Jirapinyo P, Lee LS. Endoscopic ultrasound-guided pancreatobiliary endoscopy in surgically altered anatomy. Clin Endosc 2016;49:515-529.

16. Savides TJ, Varadarajulu S, Palazzo L. EUS 2008 working group document: evaluation of EUS-guided hepaticogastrostomy. Gastrointest Endosc 2009;69(Suppl 2):S3-S7.

17. Wang K, Zhu J, Xing L, Wang Y, Jin Z, Li Z. Assessment of efficacy and safety of EUS-guided biliary drainage: a systematic review. Gastrointest Endosc 2016;83:1218-1227.

18. Itoi T, Itokawa F, Tsuchiya T, Tsuji S, Tonozuka R. Endoscopic ultrasound-guided choledochoantrostomy as an alternative extrahepatic bile duct drainage method in pancreatic cancer with duodenal invasion. Dig Endosc 2013;25 Suppl 2:142-145.

19. Cho MK, So H, Jung K, Lee JH, Park DH. Percutaneous transhepatic biliary drainage-assisted, endoscopic ultrasound-guided hepaticoduodenostomy for isolated complete right intrahepatic duct obstruction. Endoscopy 2016;48:E317-E318.

20. Park do H. Endoscopic ultrasound-guided biliary drainage of hilar biliary obstruction. J Hepatobiliary Pancreat Sci 2015;22:664-668.

21. Park SJ, Choi JH, Park DH, et al. Expanding indication: EUS-guided hepaticoduodenostomy for isolated right intrahepatic duct obstruction (with video). Gastrointest Endosc 2013;78:374-380.

22. Oh D, Park DH, Song TJ, et al. Optimal biliary access point and learning curve for endoscopic ultrasound-guided hepaticogastrostomy with transmural stenting. Therap Adv Gastroenterol 2017;10:42-53.

23. Itoi T, Isayama $\mathrm{H}$, Sofuni $\mathrm{A}$, et al. Stent selection and tips on placement technique of EUS-guided biliary drainage: transduodenal and transgastric stenting. J Hepatobiliary Pancreat Sci 2011;18:664-672.

24. Piraka C, Shah RJ, Fukami N, Chathadi KV, Chen YK. EUS-guided transesophageal, transgastric, and transcolonic drainage of intra-abdominal fluid collections and abscesses. Gastrointest Endosc 2009;70:786-792.

25. Iwashita T, Doi S, Yasuda I. Endoscopic ultrasound-guided biliary drainage: a review. Clin J Gastroenterol 2014;7:94-102. 
26. Paik WH, Park DH, Choi JH, et al. Simplified fistula dilation technique and modified stent deployment maneuver for EUS-guided hepaticogastrostomy. World J Gastroenterol 2014;20:5051-5059.

27. Ogura T, Masuda D, Takeuchi T, Fukunishi S, Higuchi K. Liver impaction technique to prevent shearing of the guidewire during endoscopic ultrasound-guided hepaticogastrostomy. Endoscopy 2015;47:E583-E584.

28. Park do H, Lee TH, Paik WH, et al. Feasibility and safety of a novel dedicated device for one-step EUS-guided biliary drainage: a randomized trial. J Gastroenterol Hepatol 2015;30:1461-1466.

29. Park DH, Jang JW, Lee SS, Seo DW, Lee SK, Kim MH. EUS-guided biliary drainage with transluminal stenting after failed ERCP: predictors of adverse events and long-term results. Gastrointest Endosc 2011;74:12761284 .

30. Kahaleh M, Artifon EL, Perez-Miranda M, et al. Endoscopic ultrasonography guided biliary drainage: summary of consortium meeting, May 7th, 2011, Chicago. World J Gastroenterol 2013;19:1372-1379.

31. Park DH, Koo JE, Oh J, et al. EUS-guided biliary drainage with one-step placement of a fully covered metal stent for malignant biliary obstruction: a prospective feasibility study. Am J Gastroenterol 2009;104:21682174.

32. Ogura T, Yamamoto K, Sano T, et al. Stent length is impact factor associated with stent patency in endoscopic ultrasound-guided hepaticogastrostomy. J Gastroenterol Hepatol 2015;30:1748-1752.

33. Ogura T, Chiba Y, Masuda D, et al. Comparison of the clinical impact of endoscopic ultrasound-guided choledochoduodenostomy and hepaticogastrostomy for bile duct obstruction with duodenal obstruction. Endoscopy 2016;48:156-163.

34. Ogura T, Sano T, Onda S, et al. Endoscopic ultrasound-guided biliary drainage for right hepatic bile duct obstruction: novel technical tips. Endoscopy 2015;47:72-75.

35. Paik WH, Lee NK, Nakai Y, et al. Conversion of external percutaneous transhepatic biliary drainage to endoscopic ultrasound-guided hepaticogastrostomy after failed standard internal stenting for malignant biliary obstruction. Endoscopy 2017 Feb 14 [Epub]. https://doi.org/10.1055/ s-0043-102388.

36. Artifon EL, Aparicio D, Paione JB, et al. Biliary drainage in patients with unresectable, malignant obstruction where ERCP fails: endoscopic ultrasonography-guided choledochoduodenostomy versus percutaneous drainage. J Clin Gastroenterol 2012;46:768-774.

37. Khashab MA, Valeshabad AK, Afghani E, et al. A comparative evaluation of EUS-guided biliary drainage and percutaneous drainage in patients with distal malignant biliary obstruction and failed ERCP. Dig Dis Sci 2015;60:557-565.

38. Sharaiha RZ, Khan MA, Kamal F, et al. Efficacy and safety of EUS-guided biliary drainage in comparison with percutaneous biliary drainage when ERCP fails: a systematic review and meta-analysis. Gastrointest Endosc 2017 Jan 4 [Epub]. https://doi.org/10.1016/j.gie.2016.12.023. 